\title{
Anesthesia for carpal tunnel release
}

\author{
Avinash Sinha MBCHB FRCA, ${ }^{*}$ Vincent Chan MD FRCPC, ${ }^{*}$ Dimitri J. Anastakis MD MED FRCSC FACs $\dagger$
}

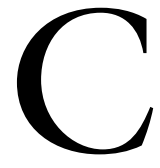

ARPAL tunnel release (CTR) involves dividing the transverse carpal ligament thereby decompressing the median nerve. The most commonly used surgical approach is the open CTR, which allows for epineurolysis of the median nerve or flexor tenosynovectomy, often required in cases with perineural scarring, associated with prolonged or recurrent symptoms. Endoscopic, minimally invasive, techniques aim to expedite the patient's recovery, by dividing the transverse carpal ligament from within the carpal tunnel, leaving the overlying structures intact. To date however, there is limited evidence to suggest open and endoscopic CTR differ significantly in this regard, although open CTR is associated with fewer complications, such as neuropraxia. ${ }^{1}$

Anesthetic options for CTR include; general anesthesia (GA), local anesthetic (LA) infiltration, intravenous regional anesthesia (IVRA), and peripheral nerve blocks (PNB), either proximally at the brachial plexus or more distally at the peripheral nerves. In this editorial, we aim to review and discuss the current literature regarding efficacy, safety, and limitations of each anesthetic option as related to optimal surgical conditions required for CTR.

The anesthetic options of choice for CTR should meet the following anesthetic and surgical requirements. A bloodless operative field, without anatomical distortion, is essential to enable clear identification of the transverse carpal ligament and median nerve, avoiding injury to the palmar cutaneous branch of the median nerve. An arm tourniquet commonly provides the bloodless field, but tourniquet pain can become intolerable, when applied for longer than $30 \mathrm{~min}^{2}$ Consistent, fast onset, surgical anesthesia, tourniquet tolerance, and prolonged postoperative analgesia are ideal, but intraoperative muscle relaxation and prolonged postoperative motor block are not required. The open CTR will be considered, but the conclusions might be equally applicable to other surgical approaches. As the extent and complexity of the surgery can exceed preoperative expectations, the anesthetic options ought to be universally applicable.

LA infiltration is the simplest, most economical technique for open CTR, providing rapid onset, prolonged anesthesia with no motor block and good patient satisfaction. ${ }^{3}$ However, the area of anesthesia does not limit tourniquet pain. Anatomical distortion due to infiltration at the site of incision is a major limitation of this technique. The local vasoconstricting effect of epinephrine when added to the LA solution avoids the use of tourniquet and infiltrating the surgical area $20 \mathrm{~min}$ before incision reduces local distortion. ${ }^{4}$ Despite this, epineurolysis or tenosynovectomy are generally not recommended. This option is necessary in those patients with contraindications to tourniquet, for example, sickle cell disease or arteriovenous fistula.

IVRA is another simple anesthetic technique, often preferred for elective outpatient hand procedures lasting under one hour. ${ }^{5}$ Rapid onset of surgical anesthesia (both sensory and motor block) is achieved after injection of $30-40 \mathrm{~mL}$ of a LA, commonly lidocaine $0.5-0.75 \%$ to a maximum of $3 \mathrm{mg} \cdot \mathrm{kg}^{-1}$. Bilateral venous cannulations, with operative limb exsanguination and tourniquet inflation are necessary. If limb exsanguination or tourniquet application is not properly performed, venous engorgement after tourniquet inflation will compromise surgical exposure. This undesirable situation cannot be remedied quickly as re-exsanguination may cause LA toxicity should the tourniquet be deflated soon after LA administration.

Recent advances in IVRA attempt to address other disadvantages of this technique: short lived post-block analgesia, potential LA systemic toxicity and tourniquet pain. Dissipation of anesthetic effect is expected soon after tourniquet deflation but postoperative analgesia can be prolonged by LA infiltration of the wound before closure. Adjuncts to IVRA, e.g., ketorolac and clonidine have been shown to improve the quality of postoperative analgesia. ${ }^{6}$ The risk of LA

From the Departments of Anesthesia* and Surgery, $\dagger$ Toronto Western Hospital, Toronto, Ontario, Canada.

Address correspondence to: Dr. Avinash Sinha, Department of Anesthesia, Toronto Western Hospital, Room EC2-046, 399 Bathurst Street, Toronto, Ontario M5T 2S8, Canada. Phone: 416-603-5118; Fax: 416-603-6494; E-mail: avi.sinha@uk.com 
toxicity has greatly diminished since safer agents replaced bupivacaine e.g., lidocaine and prilocaine. Limiting the maximum $i v$ bolus dose and keeping the minimum cuff deflation time to $15 \mathrm{~min}$, we can safely administer IVRA for CTR. Several safety strategies revolve around tourniquet application. For example, sequential cuff deflation can slow the time to peak systemic LA level during the washout period. ${ }^{7}$ Alternatively, the use of a forearm cuff for IVRA is a technique that provides effective surgical anesthesia with a LA dose reduction of up to $30 \% .^{8}$ LA leakage under the forearm tourniquet, through interosseous vessels, was initially questioned but later quantified to be similar for both forearm $(6 \% \pm 12 \%)$ and conventional upper arm cuff placement $(10 \% \pm 20 \%) .{ }^{9}$

Tourniquet pain remains the greatest limiting factor in the duration of IVRA. Double cuff tourniquets are used to prolong tolerance, by inflating the distal cuff over what is thought to be an anesthetized portion of the arm. The upper arm, adjacent to the proximal cuff, receives innervation from the intercosto-brachialis and inferio-lateral brachial cutaneous nerves. These nerves ramify into $s c$ tissues proximal to the tourniquet. Thus it is doubtful that any reliable block is achieved under the distal tourniquet portion of the double cuff. ${ }^{10}$ An area of sensitization and hyperalgesia probably exists around the painful cuff, at the site of the adjacent second cuff. ${ }^{7}$ To overcome tourniquet pain and increase tolerance, a separate forearm cuff may be applied over the area already anesthetized. This rescue technique has been found effective in a recent study. ${ }^{7}$

PNB is another anesthetic option for CTR, but is often more time consuming and requires greater technical skills. PNB may be performed proximally in the axilla where branches of the brachial plexus closely approximated. Alternatively, peripheral nerves can be blocked individually at the mid-humeral level or further distally at the elbow pericondylar level. PNB offers advantages including upper arm analgesia and tourniquet tolerance extending beyond $30 \mathrm{~min}$. There is no distortion of anatomy at the operative site since infiltration is proximal to the surgical site. Epineurolysis or tenosynovectomy can be performed safely. Additionally, PNB offers good postoperative analgesia, minimizing opioid requirement and its effects.

However, PNB results in both sensory and motor block; the latter is neither necessary nor desirable after CTR. Peripheral blocks performed at, or distal to, the elbow are attractive options as motor block is limited, because most motor nerve fibres have already ramified at this point. When anesthetizing individual peripheral nerves, it is important to point out that anesthesia over the volar aspect of the wrist and palm is required during open CTR. The median nerve predominantly innervates this area but there is sensory overlap from both ulnar and radial nerve innervation. Thus, it is advisable to block all three terminal branches of the brachial plexus. Furthermore, an incision extended proximally onto the forearm involves the cutaneous distribution of the musculocutaneous nerve. This highlights the intricacy and potential difficulty of blocking successfully all the individual peripheral nerves at the elbow.

Differential block is an attractive feature of distal PNB. It is ideal to maintain postoperative pain relief by depositing a long acting agent at the median nerve but short acting LA agents for the other terminal branches. Both mid-humeral and pericondylar PNB approaches are suitable for this and, interestingly, blocks as distal as the wrist have also been described. ${ }^{11}$

Other strategies involving the addition of opioid or $\alpha_{2}$-agonists to LA agents to prolong analgesia without associated motor block have emerged for PNB. ${ }^{12}$ Admittedly, potential disadvantages of distal PNB are longer induction time and technique related failure; however, incomplete block can be augmented with local infiltration in the operating room.

Finally, GA fulfills all the surgical requirements needed to undertake CTR. Anesthesia can be induced rapidly and maintained with no concerns of local anatomical distortion or tourniquet intolerance. Although modern GA agents are efficient and reliable, the potential complications associated with GA are not insignificant in the face of a relatively "minor" CTR procedure. Dizziness, nausea and vomiting are well known side effects in the immediate postanesthetic period. ${ }^{5,13}$ Postsurgical pain is also common but can be dealt with simply by LA wound infiltration at the end of surgery. In elderly patients, perioperative cognitive deficit is found more commonly with GA than with regional anesthesia for day surgery cases. ${ }^{14}$ Although not common, unanticipated airway difficulties and gastroesophageal reflux during GA can also result in significant morbidity.

For these reasons, many consider regional anesthetic techniques the preferred options for open CTR procedures because there is less potential for anesthetic problems. ${ }^{3,11}$ Today, complicated tertiary cases including those that require epineurolysis or tenosynovectomy can be performed under regional anesthesia e.g., IVRA. In a recent cost effectiveness study, IVRA was found to be most economical, consistently effective and associated with more rapid hospital discharge when compared to GA and PNB. ${ }^{5}$

What is the ideal anesthetic technique for CTR? In our opinion, it is the anesthetic option that is least 
invasive and has the least potential for complications. For simple open CTR, LA infiltration and IVRA are good options since they are quick and easy to perform. For complicated CTR procedures, IVRA, PNB and GA are all appropriate choices. Clearly, no one anesthetic option will meet all surgical and anesthetic requirements for CTR procedures of varying complexities. The most popular anesthetic technique for CTR varies locally from one institution to another and is dependent on the preference of surgeons, anesthesiologists and, of course, patients.

\section{L'anesthésie et la décompression du nerf médian dans le canal carpien}

La décompression dans le canal carpien (DCC) comprend la division du ligament annulaire antérieur du carpe, ce qui libère le nerf médian. La DCC ouverte, plus couramment utilisée, permet l'épineurolyse du nerf médian ou la ténosynovectomie des fléchisseurs, souvent nécessaire dans les cas de cicatrisation périneurale associée à des symptômes prolongés ou récurrents. Il y a aussi des techniques endoscopiques, minimalement effractives, conçues pour hâter la convalescence des patients. On sectionne alors le ligament annulaire antérieur du carpe, de l'intérieur du canal carpien, en gardant la structure sus-jacente intacte. Toutefois, peu de preuves favorisent actuellement la DCC ouverte ou endoscopique, les différences n'étant pas significatives, même s'il y a moins de complications, comme la neuropraxie ${ }^{1}$, associées à la DCC ouverte.

On peut choisir, pour la DCC, d'utiliser l'anesthésie générale $(A G)$, l'infiltration d'anesthésique local (AL), l'anesthésie régionale intraveineuse (ARIV) et les blocages de nerfs périphériques (BNP), soit proximal, au plexus brachial, soit distal, au niveau des nerfs périphériques. Nous passons ici en revue les publications récentes et discutons de l'efficacité, de l'innocuité et des limites des options anesthésiques au regard des conditions chirurgicales optimales pour la DCC.

Les solutions anesthésiques doivent répondre aux exigences chirurgicales et anesthésiques. Un champ opératoire exsangue, sans distorsion anatomique, est essentiel à l'identification précise du ligament annulaire antérieur du carpe et du nerf médian, ce qui peut prévenir une lésion de la branche cutanée palmaire du nerf médian. L'application d'un garrot au bras assure généralement un champ exsangue, mais la douleur causée par le garrot peut devenir intolérable après 30 min. ${ }^{2}$ Une anesthésie chirurgicale stable, à installation rapide, une tolérance au garrot et une analgésie postopératoire prolongée représentent la situation idéale, mais la myorelaxation peropératoire et le blocage moteur postopératoire prolongé ne sont pas nécessaires. Nous examinerons la DCC ouverte, bien que les conclusions puissent s'appliquer également à d'autres manoeuvres chirurgicales. L'opération pouvant être plus étendue et complexe qu'on ne s'y attendait, il faut pouvoir appliquer l'anesthésie choisie dans tous les cas.

L'infiltration d'un AL est la technique la plus simple et la plus économique pour la DCC ouverte, assurant un début rapide, une anesthésie prolongée sans blocage moteur et la satisfaction des patients. ${ }^{3}$ Mais l'aire d'anesthésie ne prévient pas la douleur du garrot. La distorsion anatomique, due à l'infiltration au site d'incision, est la principale limite de la technique. La vasoconstriction locale, causée par l'épinéphrine ajoutée à la solution d'AL permet d'éviter le garrot, et l'infiltration réalisée $20 \mathrm{~min}$ avant l'incision réduit la distorsion locale. ${ }^{4}$ Malgré cela, l'épineurolyse ou la ténosynovectomie ne sont généralement pas recommandées. Cette option demeure nécessaire dans les cas de drépanocytose ou de fistule artério-veineuse où le garrot est contre-indiqué.

L'ARIV est aussi une technique anesthésique simple, souvent choisie pour les opérations à la main, ambulatoires, réglées, de moins d'une heure. ${ }^{5}$ L'anesthésie chirurgicale (sensitive et motrice) s'installe rapidement après l'injection de $30-40 \mathrm{~mL}$ d'un $\mathrm{AL}$, habituellement de la lidocaïne à 0,5-0,75\% jusqu'à un maximum de $3 \mathrm{mg} \cdot \mathrm{kg}^{-1}$. Les cannulations veineuses bilatérales, l'exsanguination opératoire du membre et le gonflement du garrot sont nécessaires. Si l'exsanguination ou l'application du garrot ne sont pas correctement exécutées, l'engorgement veineux qui suit le gonflement du garrot pourra compromettre l'exposition de l'aire chirurgicale. Cette situation indésirable ne peut être rapidement redressée, car la réexsanguination peut causer une intoxication à l'AL si le garrot est dégonflé peu après l'administration d'AL.

Les progrès récents en ARIV tentent d'éliminer d'autres désavantages de cette technique : analgésie après-bloc de courte durée, toxicité générale potentielle de l'AL et douleur causée par le garrot. On veut que l'effet anesthésique se dissipe rapidement après le 
dégonflage du garrot, mais l'analgésie postopératoire peut être prolongée par l'infiltration d'AL dans la plaie chirurgicale avant sa fermeture. Un traitement d'appoint à l'ARIV, avec du kétorolac et de la clonidine par exemple, améliorent la qualité de l'analgésie postopératoire. ${ }^{6}$ Le risque de la toxicité de l'AL a beaucoup diminué depuis que des médicaments plus sûrs, comme la lidocaïne et la prilocaïne, remplacent la bupivacaïne. En limitant les doses maximales en bolus iv et en gardant le brassard gonflé au moins $15 \mathrm{~min}$, on peut administrer l'ARIV sans danger. Certaines mesures de sécurité entourent l'application du garrot. Ainsi, le dégonflage séquentiel du brassard peut retarder le moment du taux maximal d'AL dans l'organisme pendant l'élimination. ${ }^{7}$ Aussi, l'utilisation d'un brassard à l'avant-bras pour l'ARIV permet une anesthésie chirurgicale selon une dose réduite d'AL pouvant aller jusqu'à $30 \%{ }^{8}$ La question d'une fuite d'AL sous le garrot de l'avant-bras, par les vaisseaux interosseux, a été d'abord soulevée, puis quantifiée comme similaire, que le garrot soit placé à l'avant-bras $(6 \% \pm 12 \%)$ ou, traditionnellement, au bras $(10 \% \pm 20 \%){ }^{9}$

La douleur causée par le garrot demeure le facteur le plus limitatif de la durée de l'ARIV. Les garrots à double brassard sont utilisés pour prolonger la tolérance. On gonfle le brassard distal au-dessus d'une partie du bras présumée anesthésiée. La partie du bras adjacente au brassard proximal, reçoit l'innervation des nerfs intercosto-bracial et cutané latéal inférieur du bras. Ces nerfs se ramifient dans les tissus souscutanés près du garrot. Par conséquent, on peut se demander s'il est possible de provoquer un blocage fiable sous la partie distale du garrot du double brassard. ${ }^{10}$ Une aire de sensibilisation et d'hyperalgésie existe probablement autour du brassard douloureux, au site du second brassard. ${ }^{7}$ Pour surmonter la douleur causée par le garrot et augmenter la tolérance, un brassard séparé pour l'avant-bras peut être appliqué sur la zone déjà anesthésiée. Une récente étude a révélé l'efficacité de cette technique de secours. ${ }^{7}$

Une autre possibilité d'anesthésie pour la DCC est le BNP, mais il demande souvent plus de temps et des compétences techniques plus développées. Il peut être proximal, dans le creux axillaire, où les branches du plexus brachial sont très rapprochées. On peut également procéder au bloc d'un nerf périphérique distinct au niveau mi-huméral ou plus distal, au niveau péricondylien du coude. Le BNP présente certains avantages dont l'analgésie du bras et la tolérance au garrot qui peuvent s'étendre au-delà de $30 \mathrm{~min}$. De plus, il n'y a pas de distorsion anatomique au site opératoire, car l'infiltration est proximale au site chirurgical. L'épineurolyse ou la ténosynovectomie peuvent être réalisées en toute sécurité. Le BNP offre aussi une bonne analgésie postopératoire, ce qui réduit les besoins d'opioïdes et leurs effets.

Toutefois, le BNP provoque un blocage sensitif et moteur ; ce dernier n'est ni nécessaire ni souhaité après une DCC. Les blocs périphériques réalisés au coude, ou en aval, sont intéressants, puisque le blocage moteur est alors limité, la plupart des fibres nerveuses motrices sont déjà ramifiées à ce niveau. Quand des nerfs périphériques particuliers sont anesthésiés, l'anesthésie de la face palmaire du poignet et de la paume est nécessaire pendant la DCC ouverte. Le nerf médian innerve surtout cette zone, mais il y a un chevauchement sensitif pour l'innervation cubitale et radiale. Donc, il est recommandé d'anesthésier les trois branches terminales du plexus brachial. En outre, une incision qui s'étend en direction proximale sur l'avant-bras affecte la distribution cutanée du nerf musculo-cutané. Cette situation démontre la subtilité et la difficulté potentielle d'un blocage réussi de tous les nerfs périphériques distincts du coude.

Caractéristique attirante du BNP, le bloc différentiel est idéal pour maintenir l'analgésie postopératoire effectuée en déposant un médicament à effet prolongé au nerf médian, mais des médicaments à action brève aux autres branches terminales. Les BNP mi-huméral et péricondylaire conviennent à cette fin et, ce qui est intéressant, des blocs aussi distaux que ceux du poignet ont aussi été réalisés déjà. ${ }^{11}$

D'autres stratégies nécessitant l'addition d'opioïde ou d'agonistes $\alpha_{2}$ à des $\mathrm{AL}$, réalisées pour prolonger l'analgésie sans bloc moteur associé, sont aussi apparues dans le cadre de l'utilisation du BNP. ${ }^{12} \mathrm{Il}$ faut reconnaître que les inconvénients potentiels du BNP distal sont une induction lente et des échecs liés à la technique ; cependant, le bloc incomplet peut être augmenté en salle d'opération avec une infiltration locale.

Finalement, l'AG répond à tous les besoins chirurgicaux de la DCC. L'anesthésie peut être induite rapidement et maintenue sans crainte de distorsion anatomique locale ou d'intolérance au garrot. Même si les médicaments modernes pour l'AG sont efficaces et fiables, les complications possibles de l'AG ne sont pas sans conséquences dans le cadre d'une DCC relativement «mineure». Les étourdissements, les nausées et les vomissements sont des effets secondaires postopératoires immédiats bien connus. ${ }^{5,13}$ La douleur postchirurgicale, fréquente également, peut être traitée par une simple infiltration de la plaie avec un AL en fin d'opération. Chez les patients âgés, le déficit cognitif périopératoire est plus souvent observé avec l'AG qu'avec l'anesthésie régionale en chirurgie d'un jour. ${ }^{14}$ 
Quoique rares, les difficultés d'intubation imprévues et le reflux gastro-œsophagien pendant l'AG peuvent aussi entraîner une morbidité significative.

Pour ces raisons, nombreux sont ceux qui considèrent les techniques d'anesthésie régionale comme le meilleur choix pour une DCC ouverte, car il y a moins de problèmes anesthésiques potentiels. ${ }^{3,11}$ Aujourd'hui, des patients nécessitant des soins tertiaires compliqués, y compris ceux qui ont besoin d'épineurolyse ou de ténosynovectomie, peuvent être traités sous anesthésie régionale comme l'ARIV. Dans une étude récente sur l'efficacité des coûts, l'ARIV, comparée à l'AG et au BNP, est apparue comme la technique la plus économique, toujours efficace et associée au plus court séjour hospitalier.

Quelle est la technique anesthésique idéale pour la DCC ? Selon nous, c'est le choix anesthésique le moins effractif et qui présente le plus faible potentiel de complications. Pour une simple DCC ouverte, l'infiltration d'AL et l'ARIV sont de bons choix, puisqu'ils sont rapides et faciles à réaliser. Pour des DCC plus compliquées, l'ARIV, le BNP et l'AG sont des choix tous aussi appropriés. Aucune option anesthésique ne peut répondre à tous les besoins chirurgicaux et anesthésiques pour des DCC de complexité diverse. Et la technique anesthésique la plus utilisée pour la DCC varie d'une institution à l'autre et dépend de la préférence des chirurgiens, des anesthésiologistes et, bien sûr, des patients.

\section{References}

1 Gerritsen AAM, Uitdchaag BMJ, van Gelderc D, Scholten RJP, de Vet HCW, Bouter LM. Systematic review of randomized clinical trials of surgical treatment for carpal tunnel syndrome. Br J Surg 2001; 88: 1285-95.

2 Hutchinson DT, McClinton MA. Upper extremity tourniquet tolerance. J Hand Surg 1993; 18A: 206-10.

3 Tomaino MM, Ulizio D, Vogt MT. Carpal tunnel release under intravenous regional or local infiltration anaesthesia. J Hand Surg Br 2001; 26B: 67-8.

4 Braithwaite BD, Robinson GJ, Burge PD. Haemostasis during carpal tunnel release under local anaesthesia: a controlled comparison of a tourniquet and adrenaline infiltration. J Hand Surg Br 1993; 18B: 184-6.

5 Chan VWS, Peng PWH, Kaszas Z, et al. A comparative study of general anesthesia, intravenous regional anesthesia, and axillary block for outpatient hand surgery: clinical outcome and cost analysis. Anesth Analg 2001; 93: 1181-4.

6 Choyce A, Peng P. A systematic review of adjuncts for intravenous regional anesthesia for surgical procedures. Can J Anesth 2002; 49: 32-45.
7 Barrera-Plaza M, Peng P, Middleton B, Coleman M, Chan $V$. A new design for the double cuff tourniquet improves tourniquet tolerance during IVRA. Reg Anesth Pain Med (in press).

8 Reuben SS, Steinberg RB, Maciolek H, Manikantan P. An evaluation of the analgesic efficacy of intravenous regional anesthesia with lidocaine and ketorolac using a forearm versus upper arm tourniquet. Anesth Analg 2002; 95: 457-60.

9 Coleman MM, Peng PW, Regan JM, Chan VWS, Hendler $A L$. Quantitative comparison of leakage under the tourniquet in forearm versus conventional intravenous regional anesthesia. Anesth Analg 1999; 89: 1482-6.

10 Rosenberg $P H$. Intravenous regional anesthesia: nerve block by multiple mechanisms. Reg Anesth 1993; 18: $1-5$.

11 Delaunay L, Chelly JE. Blocks at the wrist provide effective anesthesia for carpal tunnel release. Can J Anesth 2001: 48: 656-60.

12 Murphy DB, McCartney CJL, Chan VWS. Novel analgesic adjuncts for brachial plexus block: a systematic review. Anesth Analg 2000; 90: 1122-8.

13 Chung F, Mezei G. Factors contributing to a prolonged stay after ambulatory surgery. Anesth Analg 1999; 89: 1352-9.

14 Dodds C, Allison J. Postoperative cognitive deficit in the elderly surgical patient. Br J Anaesth 1998; 81: 449-62. 